\title{
Thermodynamical length scales temperatures correlation for the Ginzburg-Landau theory
}

\author{
Z. Bousnane ${ }^{1}$, N. Merabtine ${ }^{2}$, M. Benslama ${ }^{2}$, F. Bousaad $^{1}$ \\ ${ }^{I}$ Physics Department, Faculty of Science, University of Batna, 05000 Algeria \\ ${ }^{2}$ Electromagnetism and Telecommunication Laboratory, \\ Electronics Department, Faculty of Engineering, University of Constantine, 25000, Algeria \\ E-mail:na_merabtine@hotmail.com; malekbenslama@hotmail.com
}

\begin{abstract}
The emergence of the superconducting state must obey to a variational principle representations, as considered in the classical schemes, the invariance of the extremum values, according to the use of thermodynamical functions, will suggest an internal coherence governing the jumps realized by the symmetries, against the nonunivoc determination permitted by the variations of the thermodynamical parameters. The uncertainties for macroscopic states must be associated to the existence of "macroscopicity levels", considering the interaction between cooled and cooling, the macroscopic state of each one, will be considered as a combinatory length scales interactions. The reversed role between cooled and cooling suggests that near transition point $\delta E$ will be spent in $c^{2} \Delta \Gamma$. The thermodynamical functions are obtained each from other by the uncertainty principle.
\end{abstract}

Keywords: superconductivity, length scales temperature, uncertainty principle.

Manuscript received 21.02.06; accepted for publication 23.10.06.

\section{Introduction}

We try by this essay to realize an uncertainty principles dealing with finite variation of thermodynamical parameters, in accordance with the fact that the transition will be described by the ratio of thermodynamical length scales of temperatures represented as extremums of the thermodynamical functions.

\section{Definitions}

The importance of the length scales temperatures as dealing with measurement processes was revealing that in general the macroscopic state could be considered as a combinatory length scales interactions, characterizing the critical point of transition. As defined by Landau [1]

$$
\frac{\mathrm{d} \ln T}{\mathrm{~d} \tau}=\frac{\left(\frac{\partial V}{\partial \tau}\right)_{P}}{\left(\frac{\partial Q}{\partial P}\right)_{\tau}} .
$$

The action of "cold" is formulated as converging deterministically to the appearance of complex pseudo vector, when writing,

$$
\frac{\mathrm{d} S}{\mathrm{~d} E} \sim \frac{1}{\tau}
$$

The function $T=T(\tau)$ expresses the absolute scale of the temperature. $\tau$ must be chosen in such a way that propagation of superconducting state must be seen as simple as possible, means governed by a variational principle held on $E, W, F$,

$$
(\delta E)_{S, V}=(\delta F)_{P, V}=(\delta W)_{S, P} .
$$

The indeterministic description concerning the propagation of superconducting state began by the nonexistence of the derivative as

$$
\frac{\partial T}{\partial \tau} \sim \frac{\mathrm{d} T}{\mathrm{~d} \tau} .
$$

According to this view, the relation [2]

$$
\Delta Q \Delta T \sim \hbar
$$

rises.

During the propagation, near the transition point, the deterministic description as hopped is called to assume a jump. (3b) will be rewritten as $T \Delta T \Delta S \sim \hbar$ and 
$\Delta T \Delta S \sim 7.63828 \times 10^{-12}$. This uncertainty seems informing that in the neighbourhood of transition, as to our knowledge about the temperature of the body, will be lost under a given degree of precision.

As viewed at this level, the thermodynamical scale of temperatures will not follow the law (1). It will be written:

$\frac{d \ln T}{d \tau}=\hat{F} \frac{d \ln T}{d \tau}=\hat{F} \ln T(\tau)$

and

$7.637 \times 10^{-12} \frac{d \ln T}{d \tau}=\hat{F} \ln T(\tau)$,

$\hat{F}=\hat{H}-T \hat{S}$.

$\hat{S}$ is equivalent to multiply by $\ln \Delta \Gamma$. Those considerations makes that the entropy near the transition point, will be well known when $T$ is lost. The functions $\ln T_{n}(\tau)$ are polynoms.

The situation is so, when the difference between temperatures of Helium (He) and Mercury $(\mathrm{Hg})$ in the Kammerling-Onnes experiment is in the order of $7.63828 \times 10^{-12}$. Cooling every time seems to be limited by the interaction between length scales of temperatures.

The free energy will be denoted

$F=-c^{2} \Delta \Gamma-T S$.

This law seems signifying the equivalence statistical weight-cold, which regularize the relation between the cooled and cooling. $-c^{2} \Delta \Gamma$ is expressing the reversion of cold.

Equation (4b) is rewritten as

$7.63828 \times 10^{-12} \frac{\mathrm{d} \ln T}{\mathrm{~d} \tau}=c^{2} \Delta \Gamma(\ln T(\tau))-$

$-T(\ln \Delta \Gamma)(\ln T(\tau))$.

This expression shows that the interaction between two temperature length scales $T$ and $\tau$ is governed by the potential $-T(\ln \Delta \Gamma)(\ln T(\tau))$, we write it $s(T, \tau)$. $\frac{\partial}{\partial \tau}\left[c^{2} \Delta \Gamma \ln T(\tau)\right]$ gives the momentum causing the uncertainties of $\Delta T$ on the temperature and $\Delta S$ on the entropy.

\section{The thermodynamical function expressions}

We must have the following notation

$\frac{\mathrm{d} E}{\mathrm{~d} \tau}\left(\eta(r) e^{i \phi(r)}\right)=\frac{\mathrm{d} T}{\mathrm{~d} \tau} \frac{\mathrm{d} S\left(\eta(r) e^{i \phi(r)}\right)}{\mathrm{d} \tau}$.

This expression is replaced by an expansion Series hold on $E$ and $S$ as follows $\frac{\partial E}{\partial \eta} \frac{\partial \eta}{\partial r}+\frac{\partial E}{\partial \phi} \frac{\partial \phi}{\partial r}+\left.E\right|_{\tau=\tau_{0}}=$

$=\frac{\partial T}{\partial \tau}\left(\frac{\partial S}{\partial \eta} \frac{\partial \eta}{\partial r}+\frac{\partial S}{\partial \phi} \frac{\partial \phi}{\partial r}\right)+\left.T S\right|_{T=\tau}$,

$\left.E\right|_{\tau=\tau_{0}}-\left.T S\right|_{T=\tau}=\frac{\mathrm{d} T}{\mathrm{~d} \tau}\left(\frac{\partial S}{\partial \eta} \frac{\partial \eta}{\partial r}+\frac{\partial S}{\partial \phi} \frac{\partial \phi}{\partial r}\right)-$

$-\left(\frac{\partial E}{\partial \eta} \frac{\partial \eta}{\partial r}+\frac{\partial E}{\partial \phi} \frac{\partial \phi}{\partial r}\right)$

$\left.F\right|_{T=\tau, \tau=\tau_{0}}=\left.E\right|_{\tau=\tau_{0}}-\left.T S\right|_{T=\tau}$,

which is the expression of $(\delta E)_{\tau=\tau_{0}}=\left[S(\delta T)_{\tau}+T(\delta S)_{\tau}\right]$ for $\quad(\delta E)_{\tau=\tau_{0}}=0, \quad$ we'll have $\left.\Delta F\right|_{T=\tau, \tau=\tau_{0}}=-S(\delta T)_{\tau}-T(\delta S)_{\tau}$, which implies that $\frac{\partial E}{\partial \eta} \frac{\partial \eta}{\partial r}+\frac{\partial E}{\partial \phi} \frac{\partial \phi}{\partial r}=\left.E\right|_{\tau=\tau_{0}} \cdot(\delta E)_{\tau=\tau_{0}}$ and $\left.\delta F\right|_{T=\tau, \tau=\tau_{0}}$ seems not reaching zero in the same time but will be linked by $\delta E \delta F$ as $\hbar$.

The variation of the free energy, generating propagation of order parameter, when near the transition point $\delta E$ will be spent in $c^{2} \Delta \Gamma$, meaning the existence of intrinsic states of the order parameter, where the roles between cooled and cooling are reversed.

The expressions $\Delta E \Delta F$ must mean an indetermination concerning the free energy density submitted to a length scale temperatures interaction.

\section{Conclusion}

The interaction between length scales of thermodynamical parameters, may be considered as a measurement process, inducing a non-deterministically picture, and breaks the fence between cooled and cooling, which will be expressed as the appearance of macroscopic levels for each one.

According to this, the action of cold will be limited by the levels of macroscopicity of the sample, near the transition point. It seems that the ranks of macroscopicity will depend on the standard scale $\tau$, which itself is under the action of $T$, then becoming an own results. The thermodynamical functions will have not objective picture but will be obtained as uncertainties each from other.

\section{References}

1. L. Landau, E. Lifshits, Statistical physics. Edition MIR, Moscow, 1967.

2. L. Landau, E. Lifshits, Quantum mechanics. Edition MIR, Moscow, 1967. 